\title{
PERFORMANCE MONITORING AND RESOURCE PROVISIONING IN NGN OVER ETOM
}

\author{
Priya R L and Nupur Giri \\ Department of Computer Engineering, VESIT, Mumbai, India
}

\begin{abstract}
This paper is about the implementation of performance monitoring and resource availability solution in transport stratum of Next Generation Network (NGN) over Enhanced Telecom Operations Map (eTOM) framework. Continuous monitoring of performance is done to ensure consistent Quality of Service (QoS) to the users. Monitoring of network parameters such as delay, delay variation, path unavailability, packet loss ratio is done in the transport stratum of next generation network architecture. Resource and Admission Control Functions (RACF) will interact with Management functions for performance measurement of NGN transport control traffic and transport services and allocate resources to the customers whose QoS requirements are satisfied. The above functionalities are mapped into NGN business elements, defined by eTOM framework.
\end{abstract}

\section{KEYWORDS}

Next Generation Networks, Resource availability, Quality of Service, Performance Monitoring, eTOM

\section{INTRODUCTION}

According to ITU-T [1][2] NGN definition, a next-generation network (NGN) is designed to support new services in service stratum of NGN architecture. It offers better services as per user's choice among competing service providers. To support the negotiation service resource requirements as specified in the paper [4] entitled, "Functional Architectural Design of Negotiation Service Component in NGN ITU Architecture", this paper provides a better way of resource provisioning.

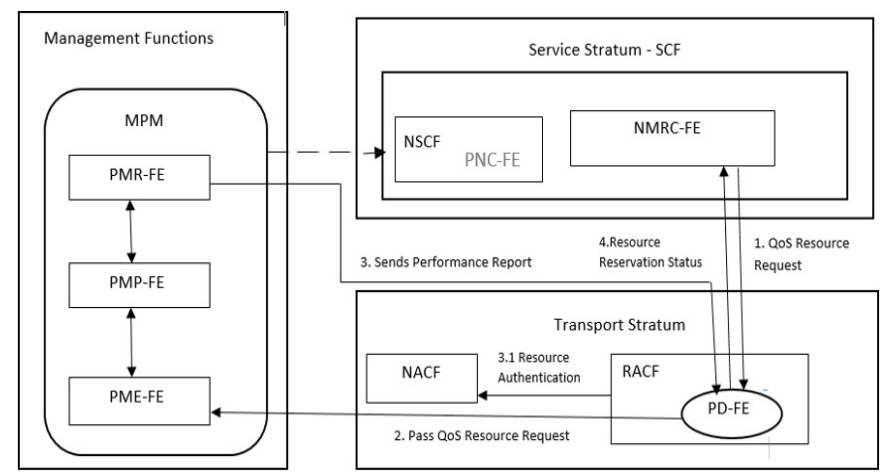

Figure 1 : Conceptual Diagram

DOI : 10.5121/ijngn.2015.7301 
International Journal of Next-Generation Networks (IJNGN) Vol.7, No.2/3, September 2015

The figure 1 specifies [4] the conceptual design of performance monitoring and resource availability solution in NGN ITU architecture. It also describes the interaction of Management of Performance Measurement (MPM) functions with components of Negotiation Service Control Functions (NSCF) and components of transport stratum in NGN.

Figure 1 show an overview of a resource availability solution as per resource request received from NMRC-FE of NSCF component. It monitors the performance of request continuously and sends performance degradation report if there exists any fall in the service network parameters and fault parameters. The components of transport stratum and management functions are illustrated as follows:

1. PD-FE (Policy Decision - Functional Entity): PD-FE component of RACF is used to determine resource availability and makes admission decision.

2. PMR-FE (Performance Measurement Reporting - Functional Entity): PMR-FE is an entity, used to send the generated performance degradation report to RACF. It does continuous monitoring of resource provisioning.

3. PMP-FE (Performance Measurement Processing - Functional Entity): PMP-FE is an entity for processing the measurement and checks the threshold values of fault parameters

4. PME-FE (Performance Measurement Execution - Functional Entity): PME-FE is an entity to perform the calculations of fault parameters and pass it to the PMP-FE.

5. NACF (Network Attachment Control Function- Functional Entity): NACF is used to authenticate the users and service providers.

6. NMRC-FE (Negotiation Resource Media Control Functional Entity): NMRC-FE is used to send the tickets after the negotiation process.

\section{FUNCTIONAL REQUIREMENTS}

Resource and Admission control functions (RACF) accepts the QoS resource request from NMRC-FE of Negotiation service control function. RACF interacts with Management of Performance Measurement (MPM) to receive status report of performance monitoring. Policy Decision Functional Entity (PD-FE) determines resource availability and makes admission decisions by enforcing policy decision rules. PD-FE generates the resource reservation ticket and passed to the service stratum component

\subsection{Non-Functional Requirements}

Application should be reliable to monitor the service parameters. The Application should be scalable to handle the increasing load of resources. Application can be run on different platforms with essential security features and isolation of processes.

\section{Proposed WORK}

\subsection{Mapping of transport stratum and management function components over eTOM level 3 Model}

Figure 2 depicts the mapping of NGN components illustrated for Performance monitoring and resource provisioning with the business elements defined over eTOM framework. 
International Journal of Next-Generation Networks (IJNGN) Vol.7, No.2/3, September 2015

\subsubsection{Mapping of PME-FE}

PME-FE is mapped with "Monitor Resource Performance" element defined by eTOM. This business element is responsible to receive resource performance information and undertakes firstin detection.

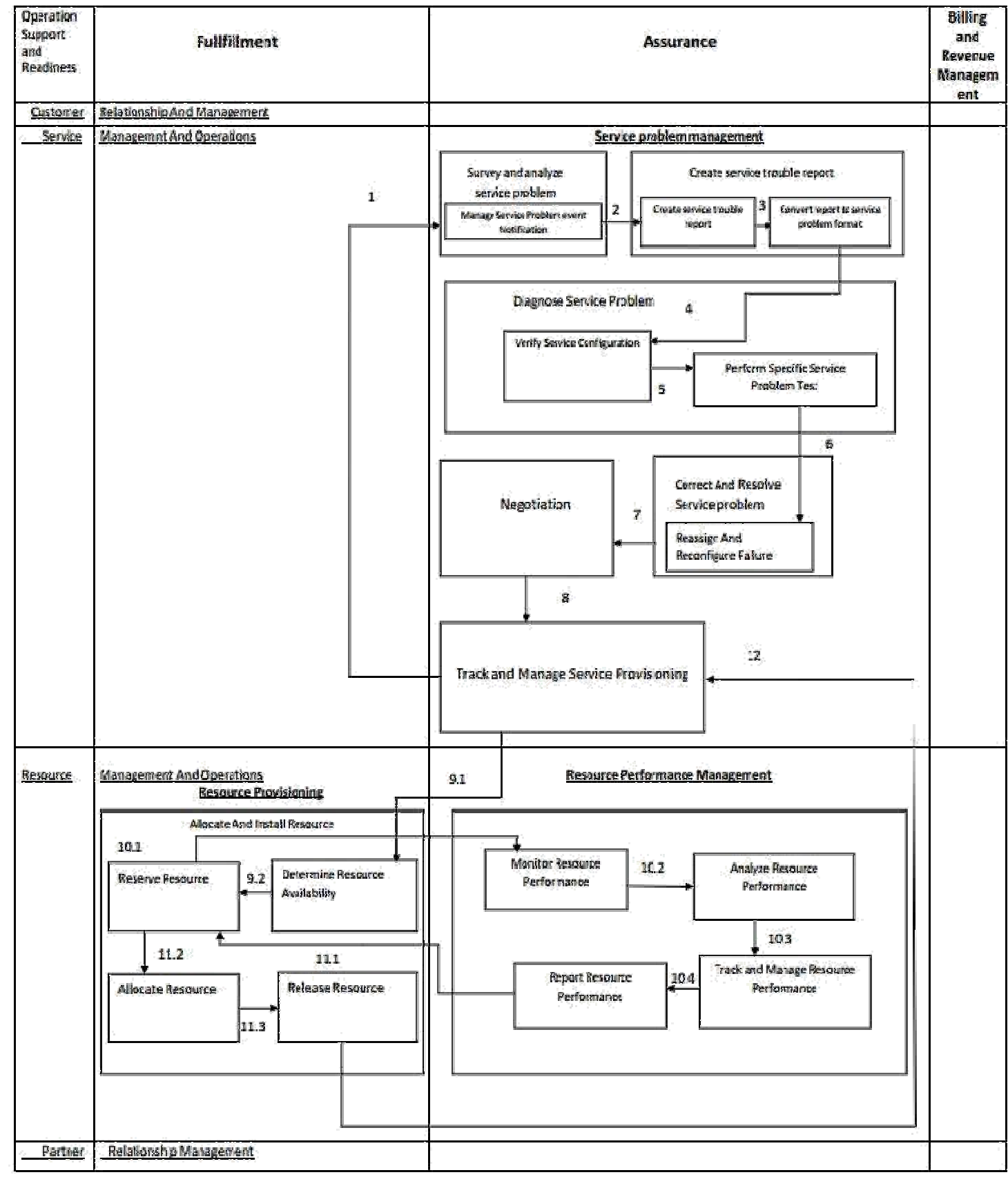

Figure 2 : eTOM Mapping 
International Journal of Next-Generation Networks (IJNGN) Vol.7, No.2/3, September 2015

\subsubsection{Mapping of PMP-FE}

PMP-FE is mapped with two business processes such as "Analyze Performance" and "Track and Manage Performance". Analyze performance element is used to analyze and evaluate the performance of specific resources. Track and Manage Performance business element ensure assignment of various activities like testing, repair and restoration. These activities are coordinated and tracked efficiently. Finally, escalation is invoked as required for any open resource performance degradation reports.

\subsubsection{Mapping of PMR-FE}

PMR-FE is mapped with "Report Resource Performance" process as defined by eTOM. It monitors the status of resource performance degradation reports. It is responsible to provide notifications about the changes. It also provides management reports for resource allocation and reservation.

\subsubsection{Mapping of NMRC-FE}

NMRC-FE is mapped with eTOM business process, "Track and Manage Service Provisioning". It determines the availability of service orders and then forward request to activate the specific services. It also requests for issuing resource orders, testing of specific services and receives test result.

\subsubsection{Mapping of PD-FE}

PD-FE is mapped with four business processes such as "Allocate, Reserve and Release Resource" and "Determine Resource Availability" defined in Resource Management and Operations layer of eTOM model. It is responsible to allocate request resources required to support a specific service. Then it investigates the ability to service specific request through feasibility check. Based on the results, resources are allocated and released to specific service order.

\section{IMPLEMENTATION}

The proposed system uses various network parameters such as delay, availability, bandwidth and capacity. The performance parameters considered for implementation are Mean Time to Repair (MTTR), Mean Time between Failures (MTBF), percentage of fault repaired by next working day (FAULTUN), percentage of fault repaired within three days in urban areas (FAULTIN), percentage of fault repaired within five days in rural areas (FAULTFIV). The threshold values are decided based on TRAI report reference as citation [3]. When the network parameters goes beyond threshold values, management functions initiates negotiation service.

\section{RESUltS AND ANALYSIS}

Figure 3 represents flow chart, describing the implementation of proposed work. The work proposes four interfaces namely : First, Rs interface, that handles QoS resource request from 
International Journal of Next-Generation Networks (IJNGN) Vol.7, No.2/3, September 2015

NMRC-FE to PD-FE and notifying resource reservation status from PMR-FE to NSCF. Secondly, Rm interface which passes QoS request to PME-FE Interface. Third, Mu interface sends performance information report generated by PMR-FE periodically to PD-FE. Lastly, Ru interface, makes RACF to interact with NACF to retrieve user authentication information.

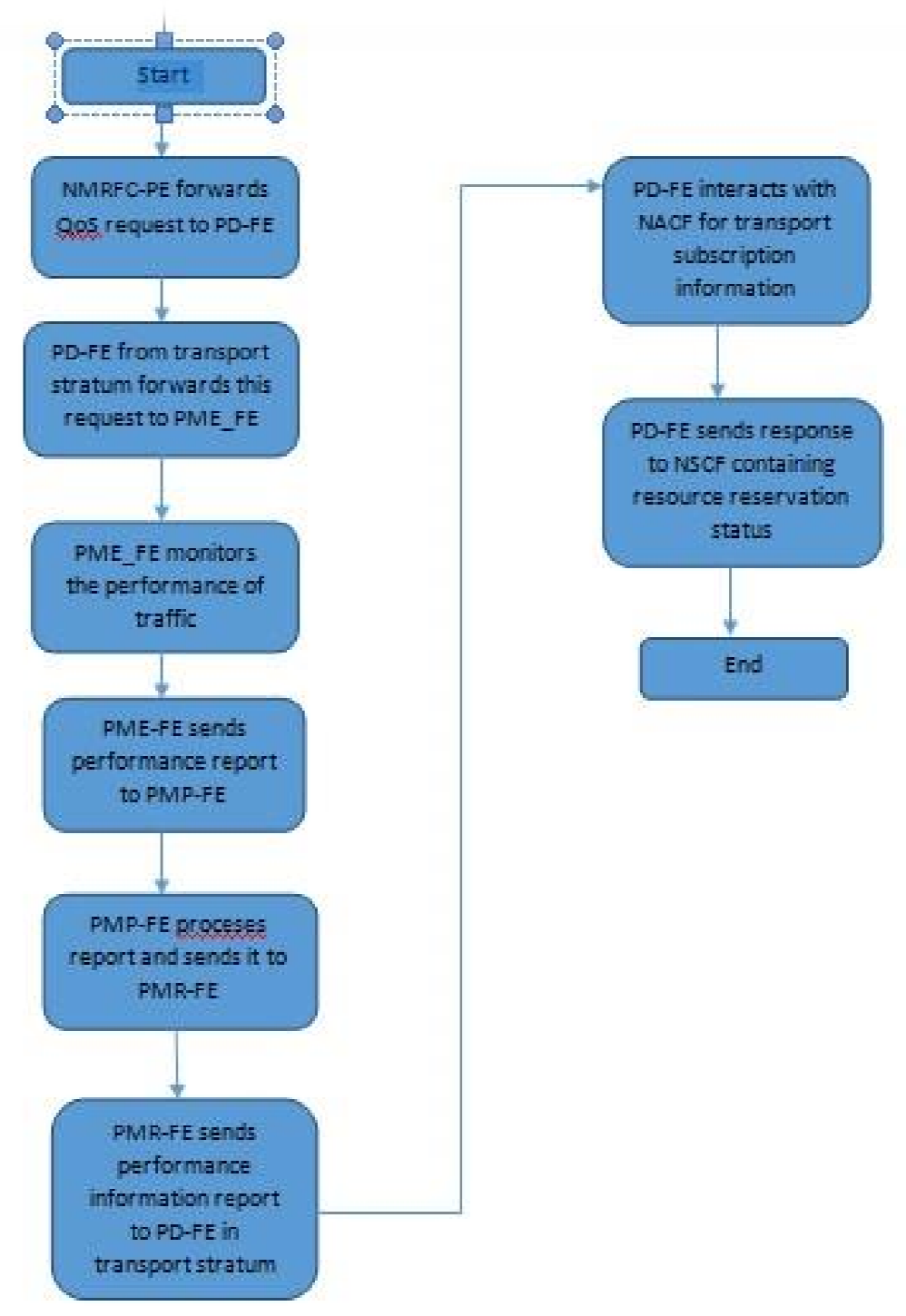

Figure 3 : Flow chart of Performance Monitoring and Resource Provisioning

The functional requirements as suggested in section 2 is accomplished in the following ways. NMRC-FE defined in Negotiation Service Control Function (NSCF) of Service Stratum is responsible to forward QoS resource request to RACF of transport stratum for the winner service provider. RACF then collects resource request from NMRC-FE to allocate resources to users. NACF checks the authentication of users in request from user database. 
International Journal of Next-Generation Networks (IJNGN) Vol.7, No.2/3, September 2015

Figure 4 shows the working of NMRC-FE, by displaying the entire received requests from service stratum of NGN. In RACF, PD-FE determines the availability, capacity, delay and bandwidth of service providers whether they can satisfy users requirements. If a particular user request cannot be satisfied, request is sent again for negotiation. RACF then passes the request to management function for monitoring of resources.

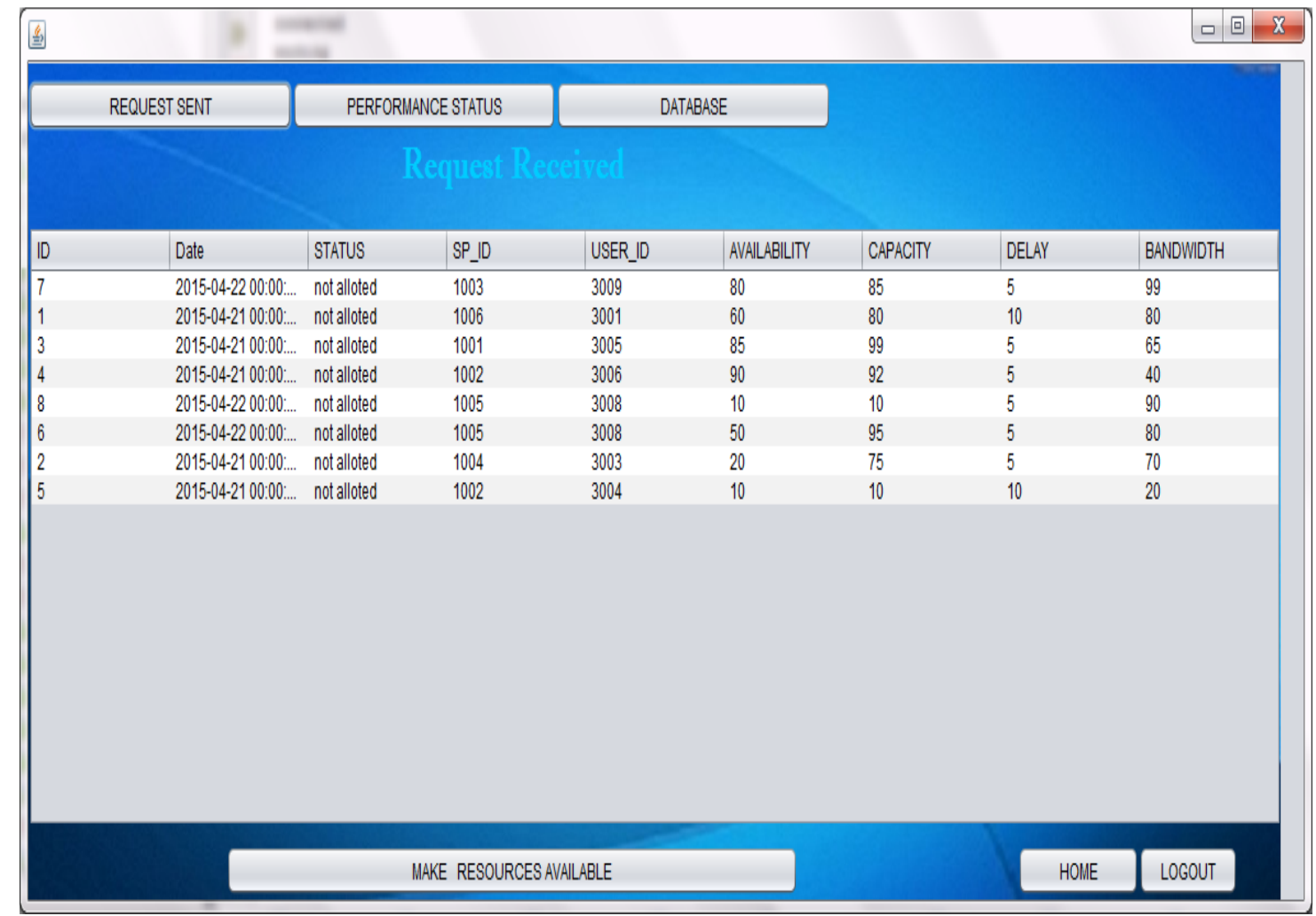

Figure 4 : NMRC - FE

PME-FE calculates MTTR,MTBF, FAULTUN, FAULTIN and FAULTFIV parameters from availability of resources and passes this to PMP-FE. PMP-FE then compares the network parameters against its lower bound and upper bound values .Users whose fault parameters does not lie between upper and lower bound ranges are sent for initiating negotiation service. Finally, PMR-FE sent the performance report to PD-FE. 
International Journal of Next-Generation Networks (IJNGN) Vol.7, No.2/3, September 2015

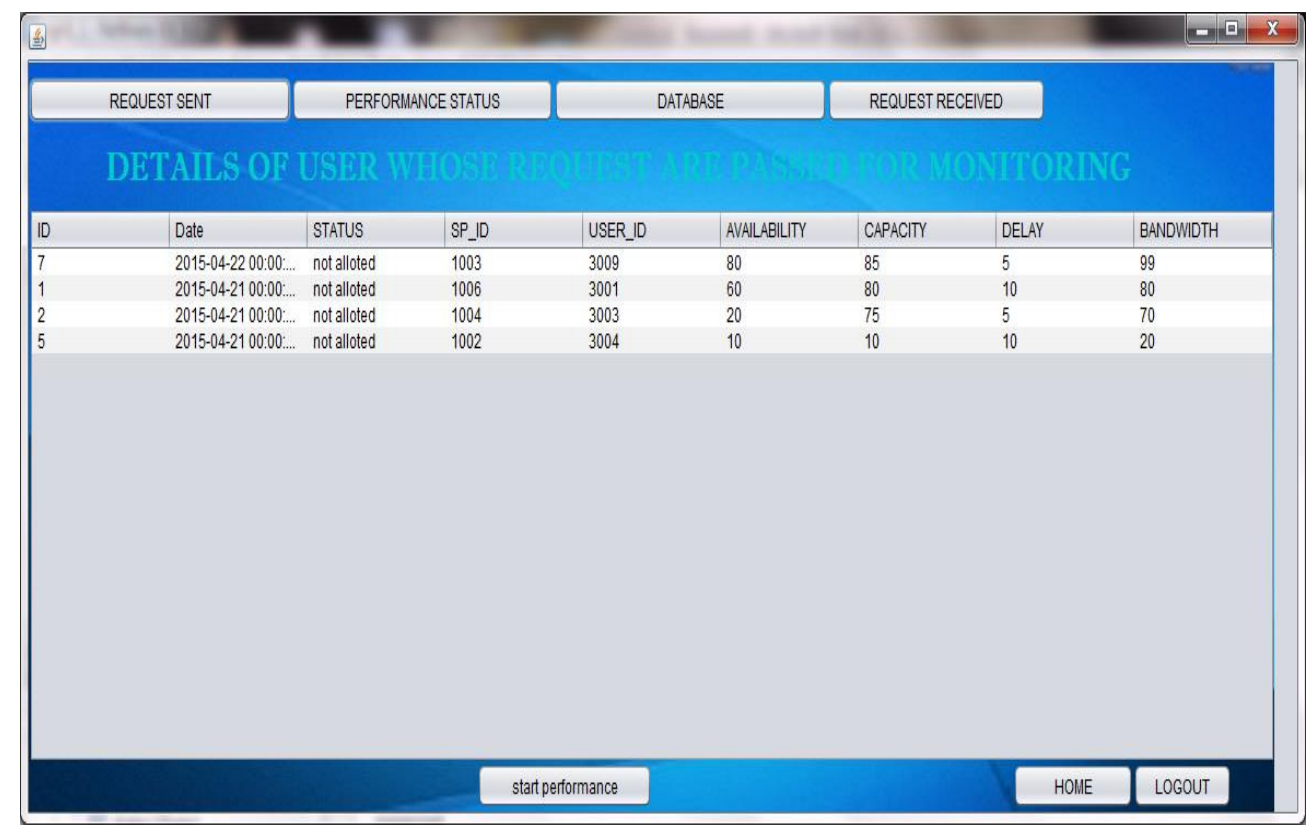

Figure 5 : MPM

Figure 5 shows the working of MPM which takes request from RACF. It further checks fault parameters and send report to PD-FE. PD-FE allocates the resources to users. Figure 6 shows working of PD-FE and final list of users to which resources are allocated.

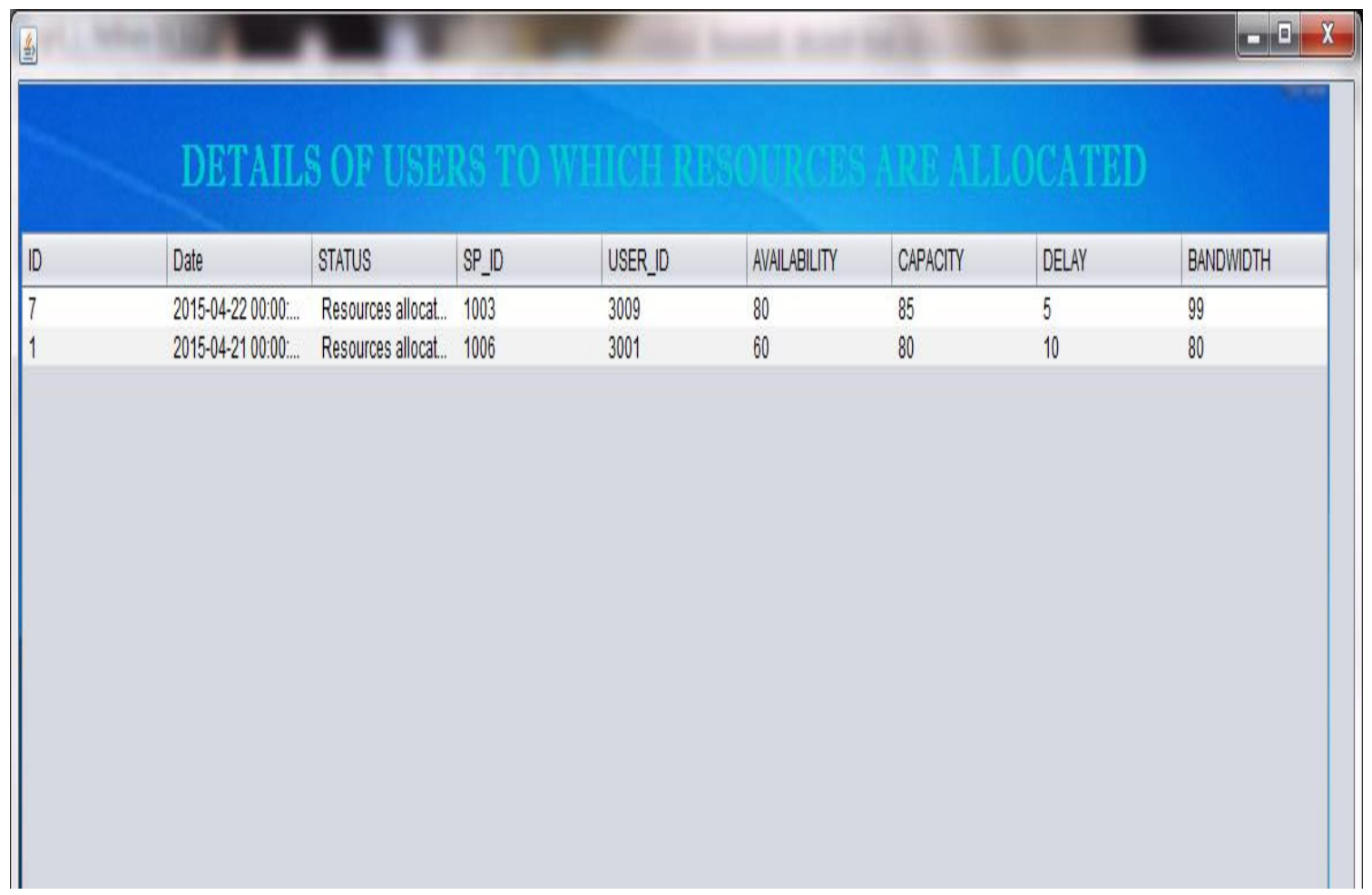

Figure $6:$ PD - FE 
Figure 7 shows working of entire dynamic process in Jade Sniffer. Jade Sniffer is a distributed platform for multi-agent interactions in telecommunication area.

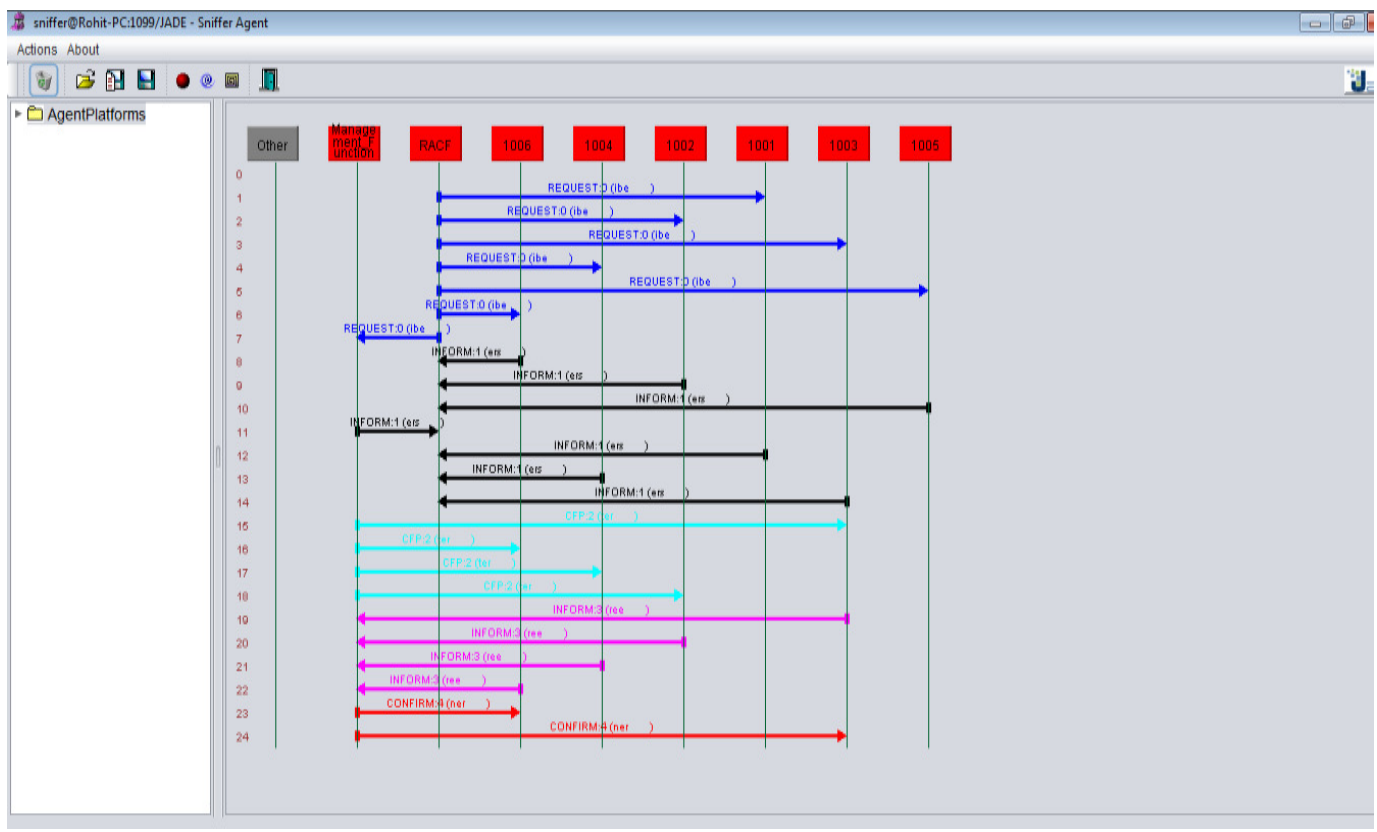

Figure 7 : Jade Sniffer Output

\section{CONCLuSions}

This paper maps all the proposed components of Performance Monitoring and Resource Provisioning in NGN architecture over eTOM level 3 model. The work focuses on to allocate the resources for those customers whose QoS requirements are fulfilled. It continuously monitor the performance and if degradation of performance occur, then it initiates for negotiation service . Thus the system ensures to provide better QoS to their cellular customers through performance monitoring and resource provisioning.

\section{REFERENCES}

[1] ITU-T Y.2011, 'General principles and general reference model for next generation networks'.

[2] ITU-T Y.2012: 'Functional requirements and architecture of the NGN'.

[3] Bapu Kabugade, Mohit Shroff, Nehil Shah, Srikant Panda and Dr. Nupur Giri, "Delay analysis of MAS based negotiations for cellular service providers" - Third International Conference on Computational Intelligence and Information Technology - CIIT 2013 OCT 18-19, 2013 in Mumbai, India

[4] Priya R.L, Dr. Nupur Giri, "Functional Architectural Design of negotiation service component in NGN ITU Architecture", International conference on telecommunication technology and Management (ICTTM) IIT Delhi (ISBN: 9780992680053), April 11-12, 2015.

[5] Enhanced telecom operations map [Website]. Retrieved from https://www.tmforum.org/standards/ business-process-framework

[6] Recommendations / RFC.Rec.ITU-T-Y.2173 
International Journal of Next-Generation Networks (IJNGN) Vol.7, No.2/3, September 2015

\section{AUTHORS}

Mrs. Priya R. L, Assistant Professor, V.E.S Institute of Technology, having 11 years of teaching experience for undergraduate students of Computer Engineering and Information Technology disciplines in different Engineering Institutes, was obtained her Master degree in Engineering from Mumbai University. Also, She had worked as a Software Engineer in different firms in Chennai and Mumbai for 4 years. Her research interest is more into Web engineering, Software engineering and Next Generation Networks

Dr. Nupur Giri, Professor \& Head of Computer Engineering department, V.E.S Institute of Technology, having more than 20 years of teaching experience for undergraduate and postgraduate students of Computer Engineering and Information Technology disciplines in VESIT, was obtained her Ph.D degree from SNDT, Mumbai. Her Ph.D research work was about the studies of Multi-Agent System in Mobile computing for Mobile Applications . Her research interest is more into Mobile Computing, Distributed computing and Next Generation Networks
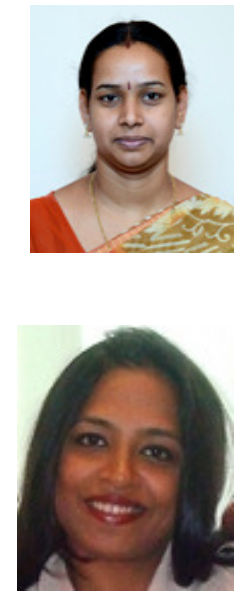